\title{
Evolution of enhanced recovery program in French university hospitals: a before-after retrospective survey
}

Hakim Harkouk

Assistance Publique - Hopitaux de Paris

Perrine Capmas

Assistance Publique - Hopitaux de Paris

Nawal Derridj

Assistance Publique - Hopitaux de Paris

Anissa Belbachir

Assistance Publique - Hopitaux de Paris

\section{Lionelle Nkam}

Assistance Publique - Hopitaux de Paris

\section{Philippe Aegerter}

Universite Versailles Saint-Quentin-en-Yvelines UFR Simone Veil sante

\section{Eva Battaglia}

Assistance Publique - Hopitaux de Paris

\section{Laure Tharel}

Assistance Publique - Hopitaux de Paris

Dominique Fletcher ( $\nabla$ dominique.fletcher@aphp.fr)

Assistance Publique - Hopitaux de Paris https://orcid.org/0000-0001-6157-1331

\section{Research}

Keywords: surgery, enhanced recovery, length of stay, post-operative complications

Posted Date: September 8th, 2020

DOl: https://doi.org/10.21203/rs.3.rs-70728/v1

License: (c) (i) This work is licensed under a Creative Commons Attribution 4.0 International License. Read Full License 


\section{Abstract}

Background: Enhanced recovery program (ERP) after surgery needs development in Assistance Publique Hôpitaux de Paris.

Methods: a retrospective before-and-after study was performed in 2015 and 2017 on three surgical models (total knee arthroplasty (TKA), colectomy, and hysterectomy) in 17 hospitals including 29 surgical departments. For each elective surgery, data were collected in one control intervention (Total hip arthroplasty (THA), gastrectomy and ovariectomy). To favour development of ERP strategy, in 2016, institutional mobilization developed with one day meeting of information, development of a Massive Open Online Course on ERP, diffusion of national update on ERP by Haute Autorité de Santé and inclusion in a regional professional partnership program. Primary outcomes were length of stay (LOS) and complications after surgery. Data on ERP items were collected in the patients' chart and in anaesthetist and surgeon interview. $70 \%$ application rate reflects application of ERP procedure.

Results: 1321 patient's files were analysed (812 in 2015 and 509 in 2017). The length of stay (LOS, mean (SD)) is reduced by 1.6 day for TKA (2015: 8.7 (6.7) versus 7.1 (3.4) in 2017; $p<0.001$ ) but increases of 0.5 day for THA (2015: 8.2 (4.1) versus 8.7 (15.5) in 2017; $p=0.011)$. LOS is stable for colectomy and hysterectomy as in their control groups (i.e. gastrectomy and ovariectomy). Incidence of severe complications after surgery is unchanged in all type of surgical models except in THA patients $(11.1 \%$ in 2015 and $1.5 \%$ in 2017, $p=0.029$ ). For TKA and hysterectomy respectively applied items of ERP (i.e: $>70 \%$ application) increased respectively from 5 to 7 out of 17 and 16 in 2015 and 2017. For colectomy, they were stable at 6 out of 21 in 2015 and 2017. All centres progressed in ERP between 2015 and 2017 for TKA ( $7 / 7$ centres) and hysterectomy ( $6 / 6$ centres). The LOS was negatively correlated with ERP items application when data collected in 2015 and 2017 were analyzed together.

Conclusion: ERP application improved between 2015 and 2017 for three surgical models after an institutional information and diffusion of recommendations in 29 surgical departments of seventeen French University hospitals

\section{Introduction}

Enhanced recovery program (ERP) for surgical patients is an approach for perioperative multimodal management of the patient aiming at a rapid restoration of previous physical and mental capacities allowing the reduction of perioperative morbidity. It also results in shorter hospital stays. This approach corresponds to a specific organization of care around patient-centered clinical paths. A recent metaanalysis confirms its effectiveness in reducing the length of stay $(-1.14$ days on average, $95 \% \mathrm{Cl}-$ 1.45 days to -0.85 days) and the overall rate of complications (risk ratio (RR) $0.71,95 \% \mathrm{Cl} 0.60$ to 0.86 ), all specialties combined (1). However, several authors point out the difficulties or failures of the method. These guidelines remain difficult to adopt essentially because they require the simultaneous and complex involvement of all members of the perioperative team (2). A Canadian team, having deployed an ERP on 
colonic surgery in more than 2 years in 15 academic hospitals, concludes to the importance of a demand that emerges from the field and the need for the cohesion of the institution (3). However, other organizational aspects are to be credited with this approach: the benefits observed, in particular in terms of care load, would allow the movement to be maintained and there is a diffusion effect towards other interventions initially not concerned (2).

In 2016, the French Haute Autorité de Santé has proposed an update in the form of an orientation report and a memo sheet encouraging the development of the ERP (4), while the Assistance Publique Hôpitaux de Paris (APHP) was mobilizing as institution to develop ERP in 2016. The evaluation of dissemination and appropriation of multi-specialty recommendations in a large group of teaching hospital would be a first in France. The use of innovative means such as an open online training site (MOOC, Massive Open Online Course) can make it easier to reach all the stakeholders concerned to promote generalization of organizational innovations.

Thus, the primary objective of this study is to measure the effects on the length of hospital stays and on safety of the implementation of ERP, throughout several hospitals in the APHP institution.

\section{Methodology}

\section{Population}

Inclusion criteria: adult patient, without opposition to the extraction of data from her/his file and the follow-up on day 30 , scheduled for one surgical model interventions or a control intervention. The following interventions were studied as ERP models since specific guidelines exist for ERP in these models, they are frequent ( $\geq 600$ annual stays at the APHP) and carried out in around ten different services (which will allow an analysis of the determinants at the service level. We chose total knee arthroplasty (approximately 1,500 interventions per year on 12 sites, 10 of which are active (i.e $>30$ interventions / year), left colectomy for cancer or non-cancer pathology (600 annual interventions on 17 sites, 9 of which have an activity > 30 interventions / year) and hysterectomy (approximately 570 procedures per year on 16 sites, 12 of which have an activity $>30$ procedures / year). This audit covered both the target intervention (ERP interventions) and control interventions of the same severity not specifically targeted by the ERP approach (i.e. having not benefited from specific clinical paths). Three other surgical models were analysed as control to observe spontaneous evolution of medical process: total hip arthroplasty for orthopaedic surgery; gastrectomy for visceral surgery and ovariectomy for gynaecological surgery

Each selected centre had more than 30 interventions per year for the selected surgical model and 30 randomly selected files were analysed per centre (20 files for ERP interventions and 10 for control interventions)

Non-inclusion criteria: none 


\section{Intervention}

The comparison scheme was "before-and-after" type for the two 2015-2017 periods comparison. We consider the year 2015 as a "reference" year and the year 2017 as a year of full implementation, 2016 being for launching.

Between the two evaluations period, an institutional awareness-raising phase on ERP in 2016 was composed of 5 steps: 1. One day of sensibilization on ERP in the institution in April 2016 with a meeting of 200 health care providers and institutional stake holders; 2. Development of a Massive Open Online Course on ERP in July 2017; 3. Diffusion of national update on ERP by Haute Autorité de Santé in June 2016 to all surgical and anaesthesia departments of APHP (4); 4. Institutional access to GRACE group in 2016 for all surgical and anaesthesiology departments offering access to guidelines, scientific literature and possibility to organize survey and benchmarking (5); 5 . Preparation of participation to a regional program organized by Regional Health Agency (Agence Régionale de Santé) offering inter institutional collaboration on ERP program. This was the initiative of ten surgical departments out of 29 involved in the survey participate in 2017 to the Regional Health Agency training program offering inter institutional collaboration on ERP program. These centres were involved as learning centres or teaching expert based on the existence of ERP protocols and previous evaluation with GRACE group. Four participate in orthopaedic surgery ( 2 expert, 2 learning centres), 3 in visceral surgery (1 expert, 2 learning) and 3 in gynaecological surgery (1 expert, 2 learning).

\section{Outcomes}

- Patients

The main demographic (age, sex), clinical (main pathology, comorbidities, ASA score, autonomy) and sociologic (living alone) characteristics were collected simultaneously. The Charleson score was calculated for all patients.

- Length of stay, complications an rehospitalisation

Patients' files were used to obtain duration of stay and incidence of complications after surgery. A list of eight complications was used for identification in the patient's file (i.e. transfert to intensive care unit, new surgery, bleeding, infection requiring antibiotic, pulmonary embolism or venous thrombosis, allergy, aggravation of existing medical condition, other). It was defined in the patient's chart whether this complication has prolonged the hospital stay or if a new hospitalization in the same institution occurred within 30 days after surgery.

\section{- Patient management and ERP}

For each ERP model intervention, a specific grid was designed based on existing ERP guidelines in 2016 for each surgery to collect data describing the patient management process pre, per and postoperatively 
(6). The cut off value of $70 \%$ for ERP item application (i.e. $70 \%$ of a particular item is applied on the patient population) was considered as reflecting sufficient appropriation (7).

- Interview of professionals

It was performed when research assistant visit the hospital for data collection with a representative of surgical and anaesthesiology department to describe level of development of ERP, resources issue and difficulties. Concerning services, the characteristics collected concerned: structure (equipment) and resource (personnel, qualification) data; commitment to quality improvement approach (Morbi mortality review; quality professional improvement), existing clinical pathways. The same independent dedicated staff trained to data collection, collected all data and performed interview in each centre

\section{Statistical analysis}

Primary outcome was the length of stay of the different surgical models selected, over a 2 periods of one year, before (year 2015) then after (year 2017) the institutional awareness-raising phase on ERP. The secondary objectives were: effects on the length of hospital stays in each centre following an awareness phase on the practice of ERP; evaluation of the conformity of practices with regard to ERP before and after awareness-raising phase; evaluation of the incidence of 30 days post-intervention complications, in each intervention, before and after the implementation of the program

We postulated that the implementation of the ERP would modify the compliance rate from $60 \%$ of noncompliant files "before" to $40 \%$ of non-compliant files "after", then requiring, for a two-sided alpha risk of $5 \%$ and $80 \%$ power, 2 groups of 110 files, ie 220 files in total ( 110 before and 110 after) per model intervention.

However, there is probably a cluster effect at hospital level, all the interventions of a model being supported by a single anaesthesia service and a single surgical team, and therefore the observations of the same hospital tend to resemble each other and provide less information than the same number of independent observations. Considering the literature on cluster effect for process-related variables, we retained a 0.02 ICC (8). Then, starting from 25 files on average per hospital and model, the design effect is around 1.5, and 380 files must be studied per model intervention (190 before and 190 after) to obtain the same information amount.

The number of "control" interventions is halved.

All comparisons were made with Chi2 test or Fischer test for discrete variable and t-test or Wilcoxon ranksum test for continuous variable between 2015 and 2017 period for the whole population and each centre for control and ERP surgical models.

A linear mixed model was used to identified variables related to LOS among patient's characteristics, by taking into account the existing correlation between files from the same surgical department. 
Missing data were analysed depending on the type of item and best available interpretation. Most of items that were not available in the patient's chart were considered as absent and non-performed.

\section{Results}

\section{Data collected}

Seventeen hospitals to participate with a total of 29 surgical and 17 anaesthesia departments involved. No data were collected in 2017 in 11 surgical departments. A total of 1321 patient's file were collected in 18 surgical departments (812 in 2015 and 509 in 2017; Table 1). Data were collected for "before" between November 2017 and June 2018 and for "after" between December 2018 and February 2020

Table 1

patients included

\begin{tabular}{|l|lll|}
\hline \multicolumn{1}{|l|}{ Type of surgery } & $\mathbf{2 0 1 5}$ & $\mathbf{2 0 1 7}$ \\
\hline Total knee arthroplasty & 195 & 137 \\
\hline Colectomy & 163 & 96 \\
\hline Hystérectomie & 190 & 120 \\
\hline Total hip arthroplasty & 99 & 66 \\
\hline Gastrectomy & 69 & 32 \\
\hline Ovariectomy & 96 & 58 \\
\hline Total & $\mathbf{8 1 2}$ & $\mathbf{5 0 9}$ \\
\hline
\end{tabular}

Data are listed in Table 2. The patients are younger in 2017 only after TKA. In most surgical models, patients are living alone more frequently in 2017. 
Table 2

patients characteristics

\begin{tabular}{|c|c|c|c|c|c|}
\hline \multirow{3}{*}{$\begin{array}{l}\text { Patient's } \\
\text { characteristics }\end{array}$} & \multirow{3}{*}{$\begin{array}{l}\text { Age (years) } \\
2015 \text { / } 2017 \\
/ p^{*}\end{array}$} & \multirow{3}{*}{$\begin{array}{l}\text { Sex (male: } \\
\text { n/\%) } \\
2015 / \\
2017 / p\end{array}$} & \multirow{3}{*}{$\begin{array}{l}\text { ASA score } \\
(1,2,3,4 ; \\
n / \%) \\
2015 / 2017 \\
/ p\end{array}$} & \multirow{3}{*}{$\begin{array}{l}\text { Charlson } \\
\text { (2015 / } 2017 \text { / } \\
\text { p) }\end{array}$} & \multirow{3}{*}{$\begin{array}{l}\text { Live style: not } \\
\text { living alone (n/\%) } \\
2015 \text { / } 2017 \text { / p }\end{array}$} \\
\hline & & & & & \\
\hline & & & & & \\
\hline \multirow{4}{*}{$\begin{array}{l}\text { TKA } \\
\text { (n: 195/137) }\end{array}$} & $\begin{array}{l}70.9(10.6) / \\
67.7(11.7)\end{array}$ & $\begin{array}{l}61(31.3) / \\
36(26.3)\end{array}$ & $\begin{array}{l}1: 14(7.2) / \\
11(8.0)\end{array}$ & $\begin{array}{l}0: 5(2.6) / 6 \\
(4.4)\end{array}$ & $\begin{array}{l}98(50.3) / 39 \\
(28.5)\end{array}$ \\
\hline & $P=0.004$ & NS & $\begin{array}{l}2: 113 \\
(57.9) / 94 \\
(68.6)\end{array}$ & $\begin{array}{l}1: 12(6.2) / 17 \\
(12.4)\end{array}$ & $P<0.0001$ \\
\hline & & & $\begin{array}{l}3: 48(24.6) \\
/ 16(11.7)\end{array}$ & $\begin{array}{l}2-7: 178 \\
(91.3) / 114 \\
(83.2)\end{array}$ & \\
\hline & & & $P=0.034$ & $P=0.084$ & \\
\hline \multirow{4}{*}{$\begin{array}{l}\text { Colectomy } \\
\text { (n: 163/96) }\end{array}$} & $\begin{array}{l}61.5(14.9) / \\
60.6(14.4)\end{array}$ & $\begin{array}{l}89(54.6) / \\
47(49.0)\end{array}$ & $\begin{array}{l}1: 26(16.0) \\
/ 26(27.1)\end{array}$ & $\begin{array}{l}0: 36(22.1) / \\
22(22.9)\end{array}$ & $\begin{array}{l}93(57.1) / 34 \\
(35.4)\end{array}$ \\
\hline & NS & NS & $\begin{array}{l}2: 94(57.7) \\
/ 52(54.2)\end{array}$ & $\begin{array}{l}1: 27(16.6) / \\
22(22.9)\end{array}$ & $P<0.0001$ \\
\hline & & & $\begin{array}{l}3: 25(15.3) \\
/ 12(12.5)\end{array}$ & $\begin{array}{l}2-7: 100 \\
(61.3) / 52 \\
(54.2)\end{array}$ & \\
\hline & & & IN & $P=0.399$ & \\
\hline \multirow{4}{*}{$\begin{array}{l}\text { Hysterectomy } \\
\text { (n: 190/120) }\end{array}$} & $\begin{array}{l}52.5(11.6) / \\
51.4(11)\end{array}$ & - & $\begin{array}{l}1: 58(30.5) \\
/ 38(31.7)\end{array}$ & $\begin{array}{l}0: 99(52.1) / \\
69(57.5)\end{array}$ & $\begin{array}{l}95(50.0) / 49 \\
(40.8)\end{array}$ \\
\hline & NS & & $\begin{array}{l}2: 92(48.4) \\
/ 55(45.8)\end{array}$ & $\begin{array}{l}1: 44(23.2) / \\
24(20.0)\end{array}$ & $P=0.033$ \\
\hline & & & $\begin{array}{l}3: 5(2.6) / 7 \\
(5.8)\end{array}$ & $\begin{array}{l}\text { 2-7: } 47(24.7) \\
\text { / } 27(22.5)\end{array}$ & \\
\hline & & & NS & $P=0.643$ & \\
\hline
\end{tabular}

$\mathrm{P*}$ : Wilcoxon rank-sum tests

The main demographic (age, sex), clinical (main pathology, comorbidities, ASA score) and sociologic (living alone) characteristics

were collected simultaneously.

The Charlson score was calculated for all patients and high score (i.e. 2-7) compared with low score (i.e. 0 and 1 ) 


\begin{tabular}{|c|c|c|c|c|c|}
\hline \multirow{3}{*}{$\begin{array}{l}\text { Patient's } \\
\text { characteristics }\end{array}$} & \multirow{3}{*}{$\begin{array}{l}\text { Age (years) } \\
2015 \text { / } 2017 \\
/ p^{*}\end{array}$} & \multirow{3}{*}{$\begin{array}{l}\text { Sex (male: } \\
\text { n/\%) } \\
2015 / \\
2017 / p\end{array}$} & \multirow{3}{*}{$\begin{array}{l}\text { ASA score } \\
(1,2,3,4 ; \\
\text { n/\%) } \\
2015 / 2017 \\
/ p\end{array}$} & \multirow{3}{*}{$\begin{array}{l}\text { Charlson } \\
\text { (2015 / } 2017 \text { / } \\
\text { p) }\end{array}$} & \multirow{3}{*}{$\begin{array}{l}\text { Live style: not } \\
\text { living alone (n/\%) } \\
2015 \text { / } 2017 \text { / p }\end{array}$} \\
\hline & & & & & \\
\hline & & & & & \\
\hline \multirow{4}{*}{$\begin{array}{l}\text { THA } \\
\text { (n: 99/66) }\end{array}$} & $\begin{array}{l}68.4(14.9) / \\
66.4(14.2)\end{array}$ & $\begin{array}{l}35(35.4) / \\
27(40.9)\end{array}$ & $\begin{array}{l}1: 15(15.2) / \\
10(15.2)\end{array}$ & $\begin{array}{l}0: 11(11.1) / 8 \\
(12.1)\end{array}$ & $\begin{array}{l}51(51.5) / 20 \\
(30.3)\end{array}$ \\
\hline & NS & NS & $\begin{array}{l}2: 53(53.5) / \\
31(47.0)\end{array}$ & $\begin{array}{l}1: 16(16.2) / \\
13(19.7)\end{array}$ & $P<0.0001$ \\
\hline & & & $\begin{array}{l}\text { 3: } 21(21.2) / \\
20(30.3)\end{array}$ & $\begin{array}{l}2-7: 72(72.7) \\
/ 45(68.2)\end{array}$ & \\
\hline & & & NS & $P=0.806$ & \\
\hline \multirow{4}{*}{$\begin{array}{l}\text { Gastrectomy } \\
\text { (n: 69/32) }\end{array}$} & $\begin{array}{l}62.9(14.6) / \\
65.4(15)\end{array}$ & $\begin{array}{l}37(53.6) / \\
24(75.0)\end{array}$ & $\begin{array}{l}1: 11(15.9) \\
/ 2(6.2)\end{array}$ & $\begin{array}{l}0: 14(20.3) / 4 \\
(12.5)\end{array}$ & $\begin{array}{l}45(65.2) / 11 \\
(34.4)\end{array}$ \\
\hline & NS & NS & $\begin{array}{l}2: 35(50.7) \\
/ 14(43.8)\end{array}$ & $\begin{array}{l}1: 12(17.4) / 7 \\
(21.9)\end{array}$ & $P=0.005$ \\
\hline & & & $\begin{array}{l}3: 18(26.1) \\
/ 7(21.9)\end{array}$ & $\begin{array}{l}2-7: 43(62.3) \\
/ 21(65.6)\end{array}$ & \\
\hline & & & $P=0.045$ & $P=0.602$ & \\
\hline \multirow{4}{*}{$\begin{array}{l}\text { Ovariectomy } \\
\text { (n: 96/58) }\end{array}$} & $\begin{array}{l}48.4(15) / \\
45.9(17.6)\end{array}$ & - & $\begin{array}{l}1: 36(37.5) \\
/ 24(41.4)\end{array}$ & $\begin{array}{l}0: 53(55.2) / \\
32(55.2)\end{array}$ & $\begin{array}{l}46(47.9) / 24 \\
(41.4)\end{array}$ \\
\hline & NS & & $\begin{array}{l}2: 39(40.6) \\
/ 17(29.3)\end{array}$ & $\begin{array}{l}1: 20(20.8) / \\
14(24.1)\end{array}$ & NS \\
\hline & & & $\begin{array}{l}3: 6(6.2) / 6 \\
(10.3)\end{array}$ & $\begin{array}{l}2-7: 23(24.0) \\
/ 12(20.7)\end{array}$ & \\
\hline & & & NS & $P=0.84$ & \\
\hline \multicolumn{6}{|c|}{ P*: Wilcoxon rank-sum tests } \\
\hline \multicolumn{6}{|c|}{$\begin{array}{l}\text { The main demographic (age, sex), clinical (main pathology, comorbidities, ASA score) and sociologic } \\
\text { (living alone) characteristics }\end{array}$} \\
\hline \multicolumn{6}{|c|}{ were collected simultaneously. } \\
\hline \multicolumn{6}{|c|}{$\begin{array}{l}\text { The Charlson score was calculated for all patients and high score (i.e. } 2-7 \text { ) compared with low score } \\
\text { (i.e. } 0 \text { and 1) }\end{array}$} \\
\hline
\end{tabular}

\section{Length of stay and complications (Table 3 )}


Table 3

length of hospital stay and complications within 30 days after surgery

\begin{tabular}{|c|c|c|c|c|}
\hline $\begin{array}{l}\text { Type of } \\
\text { surgery / } \\
\text { outcome }\end{array}$ & $\begin{array}{l}\text { Length of } \\
\text { hospital } \\
\text { stay (day) }\end{array}$ & $\begin{array}{l}\text { Incidence of } \\
\text { complications within } 30 \\
\text { days after surgery (n / \%) }\end{array}$ & $\begin{array}{l}\text { Prolongation } \\
\text { of hospital } \\
\text { stay (day) }\end{array}$ & $\begin{array}{l}\text { New hospital } \\
\text { admission within } 30 \\
\text { days after surgery }\end{array}$ \\
\hline & $\begin{array}{l}2015 / / p^{*} \\
2017 / p\end{array}$ & $2015 / 2017 / p$ & $\begin{array}{l}2015 / 2017 / \\
p\end{array}$ & $2015 / 2017 / p$ \\
\hline $\begin{array}{l}\text { TKA } \\
\text { (n: 195/137) }\end{array}$ & $\begin{array}{l}8.7(6.7) / \\
7.1(3.4) \\
p<0.001\end{array}$ & $\begin{array}{l}17(8.7 \%) / 12(8.8 \%) \\
\text { NS }\end{array}$ & $\begin{array}{l}11(5.6 \%) / \\
1(0.7 \%) \\
p=0.003\end{array}$ & $\begin{array}{l}11(5.6 \%) / 2(1.5 \%) \\
\text { NS }\end{array}$ \\
\hline $\begin{array}{l}\text { Colectomy } \\
\text { (n: 163/96) }\end{array}$ & $\begin{array}{l}10.5(6.5) / \\
13.8(38.1) \\
\text { NS }\end{array}$ & $\begin{array}{l}18(11 \%) / 11(11.5 \%) \\
\text { NS }\end{array}$ & $\begin{array}{l}7(4.3 \%) / 8 \\
(8.3 \%) \\
\text { NS }\end{array}$ & $\begin{array}{l}5(3.1 \%) / 5(5.2 \%) \\
\text { NS }\end{array}$ \\
\hline $\begin{array}{l}\text { Hysterectomy } \\
\text { (n: 190/120) }\end{array}$ & $\begin{array}{l}4.6(2.6) / \\
4.2(2.3) \\
\text { NS }\end{array}$ & $\begin{array}{l}9(4.7 \%) / 4(3.3 \%) \\
\text { NS }\end{array}$ & $\begin{array}{l}6(3.2 \%) / 3 \\
(2.5 \%) \\
\text { NS }\end{array}$ & $\begin{array}{l}6(3.2 \%) / 2(1.7 \%) \\
\text { NS }\end{array}$ \\
\hline $\begin{array}{l}\text { THA } \\
\text { (n: 99/66) }\end{array}$ & $\begin{array}{l}8.2(4.1) / \\
8.7(15.5) \\
p=0.011\end{array}$ & $\begin{array}{l}11(11.1 \%) / 1(1.5 \%) \\
p=0.029\end{array}$ & $\begin{array}{l}5(5.1 \%) / 1 \\
(1.5 \%) \\
\text { NS }\end{array}$ & $\begin{array}{l}3(3.0 \%) / 2(3.0 \%) \\
\text { NS }\end{array}$ \\
\hline $\begin{array}{l}\text { Gastrectomy } \\
\text { (n: 69/32) }\end{array}$ & $\begin{array}{l}17.7(17) / \\
15.7(8.6) \\
\text { NS }\end{array}$ & $\begin{array}{l}22(31.9 \%) / 9(28.1 \%) \\
\text { NS }\end{array}$ & $\begin{array}{l}15(21.7 \%) / \\
6(18.8 \%) \\
\text { NS }\end{array}$ & $\begin{array}{l}7(10.1 \%) / 3(9.4 \%) \\
\text { NS }\end{array}$ \\
\hline $\begin{array}{l}\text { Ovariectomy } \\
\text { (n: } 96 / 58)\end{array}$ & $\begin{array}{l}5.5(24.2) / \\
3.7(4.6) \\
\text { NS }\end{array}$ & $\begin{array}{l}2(2.1 \%) / 3(5.2 \%) \\
\text { NS }\end{array}$ & $\begin{array}{l}2(2.1 \%) / 3 \\
(5.2 \%) \\
\text { NS }\end{array}$ & $\begin{array}{l}1(1.0 \%) / 1(1.7 \%) \\
\text { NS }\end{array}$ \\
\hline
\end{tabular}

$\mathrm{P*}$ : Wilcoxon rank-sum tests

In orthopaedic surgery, the length of stay (LOS) was reduced by 1.6 day (2015: 8.7 (6.7) versus 7.1 (3.4) in 2017; $p$ <.001) for TKA but raised of 0.5 day for THA (2015: 8.2 (4.1) versus 8.7 (15.5) in 2017; $p=$ 0.011). It was stable for colectomy and hysterectomy as in control groups (i.e. THA, gastrectomy and ovariectomy).

The incidence of complications within the first month was unchanged in all type of surgical models except in THA patients (11.1\% in 2015 versus $1.5 \%$ in $2017, p=0.029)$. The prolongation of hospital stay related to complications was reduced in frequency after TKA.

\section{Patient management and ERP compliance}


Some ERP items were detailed in the results but treated as one single item (i.e. oral or subcutaneous thromboprophylaxis; technique of regional anaesthesia for pain control; less invasive surgical technique). Therefore the total effective item number was 17 for TKA, 21 for colectomy and 16 for hysterectomy.

- Orthopaedic surgery (Fig. 1)

For TKA, the regional anaesthesia techniques (i.e. femoral block, infiltration and adductor canal block) evolved over time and the three techniques were considered as appropriate and cumulated in one item. The applied items of ERP (i.e: > 70\% application) increased from 5 to 7 out of 17 between 2015 and 2017 . Five items of patient management had already reached optimal appropriation level in 2015 (i.e. > 70\%) and did not regress (antibiotic prophylaxis, hypothermia, regional anaesthesia) or even progressed significantly in 2017 (thrombosis and PONV prevention). Significant improvement in ERP was observed in 8 additional items (preoperative information and fasting, strengthening of the quadriceps, intraoperative exacyl, no tourniquet use, early mobilization, intravenous line discontinuation, drainage removal). Further improvements are still required for day of surgery admission. A similar profile was observed for THA with applied items of ERP (i.e: > 70\% application) increased from 5 to 7 out of 17 between 2015 and 2017 (data not shown).

- Visceral surgery (Fig. 2)

For colectomy, applied items of ERP (i.e: > 70\% application) were stable at 6 out of 21 in 2015 and 2017. Within the six items of patient management which had already reached optimal appropriation level in 2015 (i.e. > 70\%) some were stable (antibiotic prophylaxis, hypothermia, PONV) when intraoperative use of dexamethasone or no colic preparation decline and thrombosis prevention significantly increases. Significant improvement in patient management and ERP was observed for preoperative fasting, early feeding and mobilization and intravenous line discontinuation. Numerous improvements are still required on preoperative information, use of carbohydrate, same day admission, use of local anaesthetic, volemia monitoring, nasogastric tube, urinary drain and drain removal. For gastrectomy applied items of ERP (i.e: $>70 \%$ application) decline from 5 out of 21 in 2015 to 4 out of 21 in 2017.

- Gynaecological surgery (Fig. 3)

For hysterectomy, applied items of ERP (i.e: >70\% application) increased from 5 to 7 out of 16 between 2015 and 2017. Five items of patient management had already reached optimal appropriation level in 2015 (i.e. > 70\%) and were stable (intraoperative use of dexamethasone, antibiotic prophylaxis, hypothermia, PONV and thrombosis prevention). Significant improvement in patient management and ERP was observed for compression stockings, modern fasting protocol, non-invasive surgery, early feeding and mobilization, early discontinuation for vaginal lock or bladder catheter. Numerous improvements are still required on preoperative information, same day admission, use of local anaesthetic, and early discontinuation of intravenous infusion. A similar profile was observed for ovariectomy with applied items of ERP (i.e: $>70 \%$ application) increases from 6 to 7 out of 21 between 2015 and 2017. 
- Individual trajectory of different structures (Fig. 4A, 4B, 4C)

All centres with data collected at 2015 and 2017, progressed in ERP between 2015 and 2017 for TKA (7/7) and hysterectomy (6/6) with no significant difference between centres. Evolution was mixed for colectomy with some centres reducing their application (2/5) and some improving (3/5) with no significant difference between centres. For the 6 learning centres participating to the Regional Health Agency training program the trajectory was not significantly different from other centres $(p>0.99)$. The single expert centre in gynaecological surgery has the best performance on 2017; the 2 expert centres in orthopaedic surgery were the second and third best centres in 2017; in visceral surgery the only expert centre did not collect data in 2017.

\section{Correlation between patient management and outcome (Fig. 5, Fig. 6)}

All individual data of both periods before and after were analysed for relation between individual LOS and number of ERP items applied. There is a significant correlation between LOS reduction with increased application of ERP for TKA, colectomy and hysterectomy (Spearman test; $p$ value and correlation coefficient: TKA: 0.00003 and -0.202 ; colectomy: $<0.001$ and -0.232 ; hysterectomy: 0.0018 and -0.182 respectively). When grouping in three categories according to a previous publication describing a dose dose-response relationship between various level of ERP item application and LOS $(6,8)$ (i.e. ERP items application $<50 \% ; 50-70 \%,>70 \%)$, there is a significant relation for TKA $(p=0.002)$ and hysterectomy ( $p$ $=0.002) ;$ Kruskal Wallis test.

\section{Patient's characteristics impacting LOS}

In univariate analysis for all data collected in the three surgical models gender, age and comorbidities were associated with LOS. Men had a longer average length of stay than women $(p=0.0062)$; the length of stay increases by an average of 0.1 days for an increase of one year of age; the length of stay was higher for high Charlson categories. In multivariate analysis on all the data, sex and age were associated with the length of stay. Regarding the results of analyses by surgical model, only age was associated with LOS after visceral surgery (colectomy and gastrectomy) gynaecological surgery (hysterectomy and ovariectomy). Life style and ASA score were not associated with LOS.

\section{Structural and organizational evaluation with interview of professionals}

In each centre, one anaesthetist and one surgeon were interviewed regarding their perception of ERP development in the specific surgery analysed by the survey. The results in the three surgical models were similar with declaration of increased implication of health care providers in ERP between 2015 and 2017. However, compared to observed data in ERP application, the declarative information generally overestimates the level of performance. As an example the rate of specific patient information on ERP was declared as $100 \%$ when maximal observed rate was $32 \%$ for TKA. The health care providers declare that they use specific indicators to monitor ERP evaluation in 50\% of cases in 2015 and $100 \%$ in 2017. 
Despite institutional availability in all surgical and anaesthesiology departments, rate of GRACE survey tool was declared as persistently low ( $0 \%$ in 2015 and 14\% in 2017). Frequently declared encountered difficulties are lack of adherence to the ER concept and lack of multidisciplinary communication, limited manpower, specifically ERP nurse coordinator. Organization of ERP was also limited by the difficulty to organize admission on same day, lack of available time to start ERP and patients' reluctance.

\section{Discussion}

This survey in twenty-nine surgical departments located in seventeen different university hospitals of Assistance Publique Hôpitaux de Paris (APHP) describes an improvement in the development on ERP in orthopaedic and gynaecological surgery between 2015 and 2017 with correlation between ERP items application and reduction of LOS and a significant reduction of LOS for TKA surgery.

\section{LOS and postoperative complications}

We observe a significant reduction of LOS after TKA and a slight increase after THA and no modification in the other surgical models. The mean LOS in 2017 in our survey for TKA, colectomy and hysterectomy is much longer than the LOS reported presently in the literature (respectively 4 days for TKA and 2 days for colectomy) and underlines mainly the important room for improvement still existing in our institution (9, 10). Interestingly in all individual data collected in our survey in 2015 and 2017, a significant correlation exists between the number of ERP items applied and LOS. After TKA and hysterectomy, LOS showed a negative relationship when more than $70 \%$ of ERP are applied. These results are in line with previous publication supporting that increased application of ERP protocols impact favourably LOS (11).

Therefore the LOS reduction observed after TKA may partially reflect the beneficial impact of ERP items increased application. In multivariate analysis, other patient's related factors such as younger age and female gender seem associated with reduced LOS. Younger age in the 2017 population was observed only after TKA and it certainly participates in 2017 LOS reduction after TKA.

The general principle of ERP is to enhance recovery, reduce complications with potential related LOS reduction (12). In our survey the incidence of postoperative complications after TKA, colectomy and hysterectomy is comparable to what is described in the literature $(9,13-14)$. We observe a reduction of complications only after THA with surprisingly a slight prolongation of LOS. Although the reduction of complications incidence might correlate with the positive evolution in ERP application observed after THA, as described in the literature (11), the disconnection with LOS rather suggest impact of other nonmedical factors such as unavailability of recovery institution for patients after surgery or patients unwilling to be discharged from hospital. After TKA the incidence of complications was similar but prolongation of LOS related to complications was reduced suggesting a reduced severity of post-surgical complications impacting positively LOS.

\section{Evolution of ERP item application}


Some ERP items were already largely applied in 2015 and were comforted in 2017 such as non-invasive surgery for colectomy and hysterectomy, no colic preparation for colectomy, prevention of PONV and hypothermia, antibioprophylaxis, thromboprophylaxis for the three surgical models and regional anaesthesia for pain control for TKA. Among these seven items, 2 are under the responsibility of surgeon (less invasive surgery and no colic preparation) and developed for a long time before ERP concept. The five others are accessible to anaesthetist's prescription and relate to older specific recommendations such as those of the French Society of Anaesthesia and Intensive Care on antibioprophylaxis (15), PONV (16), postoperative pain control (17), thrombosis (18). This anteriority may explain why some of those items were already largely applied in 2015.

We observe a trend to improvement in ERP application in all orthopaedic and gynaecological departments. Similar changes were not observed in visceral surgery where ERP application evolution appears heterogeneous although this particular surgical model has been targeted for ERP for a long time (7). The definition of applied item of ERP (action traced in the patients' chart and percentage of application $>70 \%$ ) can be considered as a high standard explaining potentially the final low application rate (30-45\%) of EPR items across the three surgeries in 2017. This level of appropriation has been suggested in single centre cohort study and confirmed in large national survey $(7,11)$. This high ERP application is a guarantee of outcome improvement on adverse event and LOS (11). Our results are in line with the frequency of high ERP application observed in $21 \%$ of hospital in a large survey in USA (11). In addition, a larger number of ERP items progressed significantly between 2015 and 2017 reflecting extensive significant on-going progress in ERP application. This positive evolution probably explains the significant correlation in all individual data collected between ERP application and reduction of LOS. When compared with TKA, colectomy and hysterectomy groups, no significant differences appear in the evolution of ERP application in control surgical groups. All together these results support the hypothesis of a common evolution in all involved centres of APHP institution potentially not related to the sensitization period in 2016.

Between 2015 and 2017 some items improved slightly or not at all and deserve further dissemination across all surgical models. Emerging pre-habilitation and preoperative information for TKA are still limited in 2017 (respectively $14.6 \%$ and $32.1 \%$ ) and totally absent for colectomy and hysterectomy. Although the benefit of pre-habilitation is still debated $(19,20)$, preoperative patient information is crucial to prepare and follow up an ERP (21). The absence of dedicated personnel described in surgeon and anaesthesiologist interview may participate to this lack of preoperative information strategy (21). Modern fasting rules are slightly improving (20-40\% in 2017 vs $0 \%$ in 2015), but carbohydrates which can significantly improve patient outcome after visceral surgery is still completely absent before colectomy (22). Although the absence of tourniquet for TKA surgery improved, it is still present for half of the patients (50\% vs $17 \%$ ). Early feeding on the day of surgery has progressed in 2017 (i.e. $98.4 \%$ for TKA, $86.4 \%$ for hysterectomy) but still half of patient do not benefit of this approach after colectomy. Some efficient techniques for pain control using local anaesthetic in 2017 are widespread in orthopaedic surgery but still rare for hysterectomy and slightly declining for colectomy. These analgesic techniques using regional anaesthesia have a positive impact of on ERP outcomes after colorectal surgery and 
should be used (23). Finally early mobilization which is widely mentioned in guidelines for ERP although its direct benefit on recovery and LOS is still debated (24), has developed but is still limited to half of patient on the day of surgery in 2017.

The reasons for these low implementation rates remain speculative and certainly multifactorial (5). Firstly, knowledge and understanding of recommendations on ERP suppose rather French guidelines and during the period of our institutional survey French recommendations on ERP were proposed only by HAS in 2016 (4) and French Societies for colorectal surgery in 2014 (6), but none on orthopaedic surgery and gynaecological surgery at the time of this survey. Secondly, our methodology to enhance ERP protocol application in 2016 did not coach individually surgical or anaesthesiology departments but rather APHP institution in general and the absence of direct coaching of health care teams probably failed to initiate significant progress in ERP application. In line with this strategy evolution, this type of personalized coaching just started in 2017 in APHP but certainly did not already produce beneficial effects at the time of survey in 2017.

\section{Limitations and strength of the survey}

This survey was able to collect a large sample of patients' chart analysis in seventeen different university hospitals in APHP group. The direct analysis of chart allows measurement of effective medical performance. We respect the exhaustive description of all ERP items and outcomes (LOS and adverse events) in the six surgical model with clear definition of all data collected (25).

The limitation of this survey is related to its organization only in APHP largest University French hospital limiting the generalization of the results. The other weakness is related to important reduction (38\%) of data collection in 2017 due to impossibility of 6 hospitals out of 17 participants to collect compliance data. Finally, the modifications proposed on 2016 to improve management of patient were proposed on a national (HAS recommendations, GRACE group affiliation) and institutional level (MOOC training), without personalized follow up and coaching in each hospital involved in the survey. Direct imputability of observed changes to these measures is impossible and changes are probably due to natural evolution in time as suggested by similar evolution in control surgical models (i.e THA, gastrectomy and ovariectomy)

\section{Conclusion}

In this regional survey of 29 surgical departments in 17 university hospitals in APHP it has been observed a significant improvement of ERP program application with significant reduction in LOS for TKA surgery. Further improvement will require additional training with prioritized objectives and institutional support

\section{Declarations}

Ethics approval and consent to participate: Ethic Committee approval nor individual written consent was not necessary according to French law since only already collected data were gathered. This project was 
registered (Clinical trial $N^{\circ}$ : NCT03552913) and had an agreement of the Commission Nationale Informatique et Liberté (June $6^{\text {th }}$ 2017)

Consent for publication: not applicable

Availability of data and materials:

https://www.dropbox.com/s/ycbauj9i3zo09sh/URCPO_RAAC_RapportStatistique_LNM_V05.pdf?dl=0

Competing interests: The authors declare that they have no competing interests

Funding: the project benefits for support from the Direction du Pilotage et de la Tranformation de I'Assistance Publique Hôpitaux de Paris et d'un Contrat de Recherche Clinique 2016 de l'Assistance Hôpitaux de Paris

Authors' contributions: "HH analysed and interpreted the patient data, read and approved the final manuscript. PC read and approved the final manuscript. ND organize research read and approved the final manuscript. $A B$ read and approved the final manuscript. $L N$ analysed and interpreted the patient data, read and approved the final manuscript. PA analysed and interpreted the patient data, read and approved the final manuscript. EB read and approved the final manuscript. LT read and approved the final manuscript. DF prepares and organizes research, analysed and interpreted the patient data, read and approved the final manuscript.

Acknowledgements: we want to thank the anaesthesiologist, surgeons and administrative personal of the centres which participated in the survey

\section{References}

1. Nicholson A, Lowe MC, Parker J, Lewis SR, Alderson P, Smith AF. Systematic review and metaanalysis of enhanced recovery programmes in surgical patients. Br J Surg. 2014;101(3):172-88.

2. Kahokehr A, Sammour T, Zargar-Shoshtari K, Thompson L, Hill AG. Implementation of ERAS and how to overcome the barriers. Int J Surg. 2009;7(1):16-9.

3. McLeod RS, Aarts MA, Chung F, Eskicioglu C, Forbes SS, Conn LG, et al. Development of an Enhanced Recovery After Surgery Guideline and Implementation Strategy Based on the Knowledge-to-action Cycle. Ann Surg. 2015;262(6):1016-25.

4. HAS Programmes de. réhabilitation rapide en chirurgie: état des lieux et perspectives - Note de cadrage. http://www.has-sante.fr/portail/jcms/c_1763416/fr/programmes-de-rehabilitation-rapideen-chirurgie-etat-des-lieux-et-perspectives-note-de-cadrage (last access 08042020).

5. Slim K, Delaunay L, Joris J, Leonard D, Raspado O, Chambrier C, et al. How to implement an enhanced recovery program? Proposals from the Francophone Group for enhanced recovery after surgery (GRACE). J Visc Surg. 2016;153(6S):45-S9. 
6. Alfonsi P, Slim K, Chauvin M, Mariani P, Faucheron JL, Fletcher D, et al. French guidelines for enhanced recovery after elective colorectal surgery. J Visc Surg. 2014;151(1):65-79.

7. Gustafsson UO, Hausel J, Thorell A, Ljungqvist O, Soop M, Nygren J, et al. Adherence to the enhanced recovery after surgery protocol and outcomes after colorectal cancer surgery. Arch Surg. 2011;146(5):571-7.

8. Fletcher D, Fermanian C, Mardaye A, Aegerter P, Pain, Regional Anesthesia Committee of the French $A$, et al. A patient-based national survey on postoperative pain management in France reveals significant achievements and persistent challenges. Pain. 2008;137(2):441 - 51.

9. Ripolles-Melchor J, Abad-Motos A, Diez-Remesal Y, Aseguinolaza-Pagola M, Padin-Barreiro L, Sanchez-Martin R, et al. Association Between Use of Enhanced Recovery After Surgery Protocol and Postoperative Complications in Total Hip and Knee Arthroplasty in the Postoperative Outcomes Within Enhanced Recovery After Surgery Protocol in Elective Total Hip and Knee Arthroplasty Study (POWER2). JAMA Surg. 2020:e196024.

10. Garulli G, Lucchi A, Berti P, Gabbianelli C, Siani LM. "Ultra" E.R.A.S. in laparoscopic colectomy for cancer: discharge after the first flatus? A prospective, randomized trial. Surg Endosc. 2017;31(4):1806-13.

11. Memtsoudis SG, Fiasconaro M, Soffin EM, Liu J, Wilson LA, Poeran J, et al. Enhanced recovery after surgery components and perioperative outcomes: a nationwide observational study. $\mathrm{Br} \mathrm{J}$ Anaesth. 2020;124(5):638-47.

12. Ljungqvist O, Scott M, Fearon KC. Enhanced Recovery After Surgery: A Review. JAMA Surg. 2017;152(3):292-8.

13. Greco M, Capretti G, Beretta L, Gemma M, Pecorelli N, Braga M. Enhanced recovery program in colorectal surgery: a meta-analysis of randomized controlled trials. World J Surg. 2014;38(6):153141.

14. Wenzel HHB, Kruitwagen R, Nijman HW, Bekkers RLM, van Gorp T, de Kroon CD, et al. Short-term surgical complications after radical hysterectomy-A nationwide cohort study. Acta Obstet Gynecol Scand. 2020;99(7):925-32.

15. Martin C, Pourriat JL. [Perioperative antibiotic prophylaxis practice of French anesthesiologists and resuscitators: results of a national survey]. Ann Fr Anesth Reanim. 1997;16(7):913-7.

16. Diemunsch $P$, Societe francaise d'anesthesie et de réanimation. [Conference of experts-short text. Management of postoperative nausea and vomiting. French Society of Anesthesia and Resuscitation]. Ann Fr Anesth Reanim. 2008;27(10):866 - 78.

17. Aubrun F, Nouette-Gaulain K, Fletcher D, Belbachir A, Beloeil H, Carles M, et al. Revision of expert panel's guidelines on postoperative pain management. Anaesth Crit Care Pain Med. 2019;38(4):40511.

18. Samama CM, Gafsou B, Jeandel T, Laporte S, Steib A, Marret E, et al. [French Society of Anaesthesia and Intensive Care. Guidelines on perioperative venous thromboembolism prophylaxis. Update 2011. Short text]. Ann Fr Anesth Reanim. 2011;30(12):947-51. 
19. Hoogeboom TJ, Dronkers JJ, Hulzebos EH, van Meeteren NL. Merits of exercise therapy before and after major surgery. Curr Opin Anaesthesiol. 2014;27(2):161-6.

20. Carli F. Physiologic considerations of Enhanced Recovery After Surgery (ERAS) programs: implications of the stress response. Can J Anaesth. 2015;62(2):110-9.

21. Sibbern T, Bull Sellevold V, Steindal SA, Dale C, Watt-Watson J, Dihle A. Patients' experiences of enhanced recovery after surgery: a systematic review of qualitative studies. J Clin Nurs. 2017;26(910):1172-88.

22. Nygren J, Thorell A, Ljungqvist O. Preoperative oral carbohydrate therapy. Curr Opin Anaesthesiol. 2015;28(3):364-9.

23. Mclsaac DI, Cole ET, McCartney CJ. Impact of including regional anaesthesia in enhanced recovery protocols: a scoping review. Br J Anaesth. 2015;115(Suppl 2):ii46-56.

24. Fiore JF Jr, Castelino T, Pecorelli N, Niculiseanu P, Balvardi S, Hershorn O, et al. Ensuring Early Mobilization Within an Enhanced Recovery Program for Colorectal Surgery: A Randomized Controlled Trial. Ann Surg. 2017;266(2):223-31.

25. Day RW, Fielder S, Calhoun J, Kehlet H, Gottumukkala V, Aloia TA. Incomplete reporting of enhanced recovery elements and its impact on achieving quality improvement. Br J Surg. 2015;102(13):1594602.

\section{Figures}

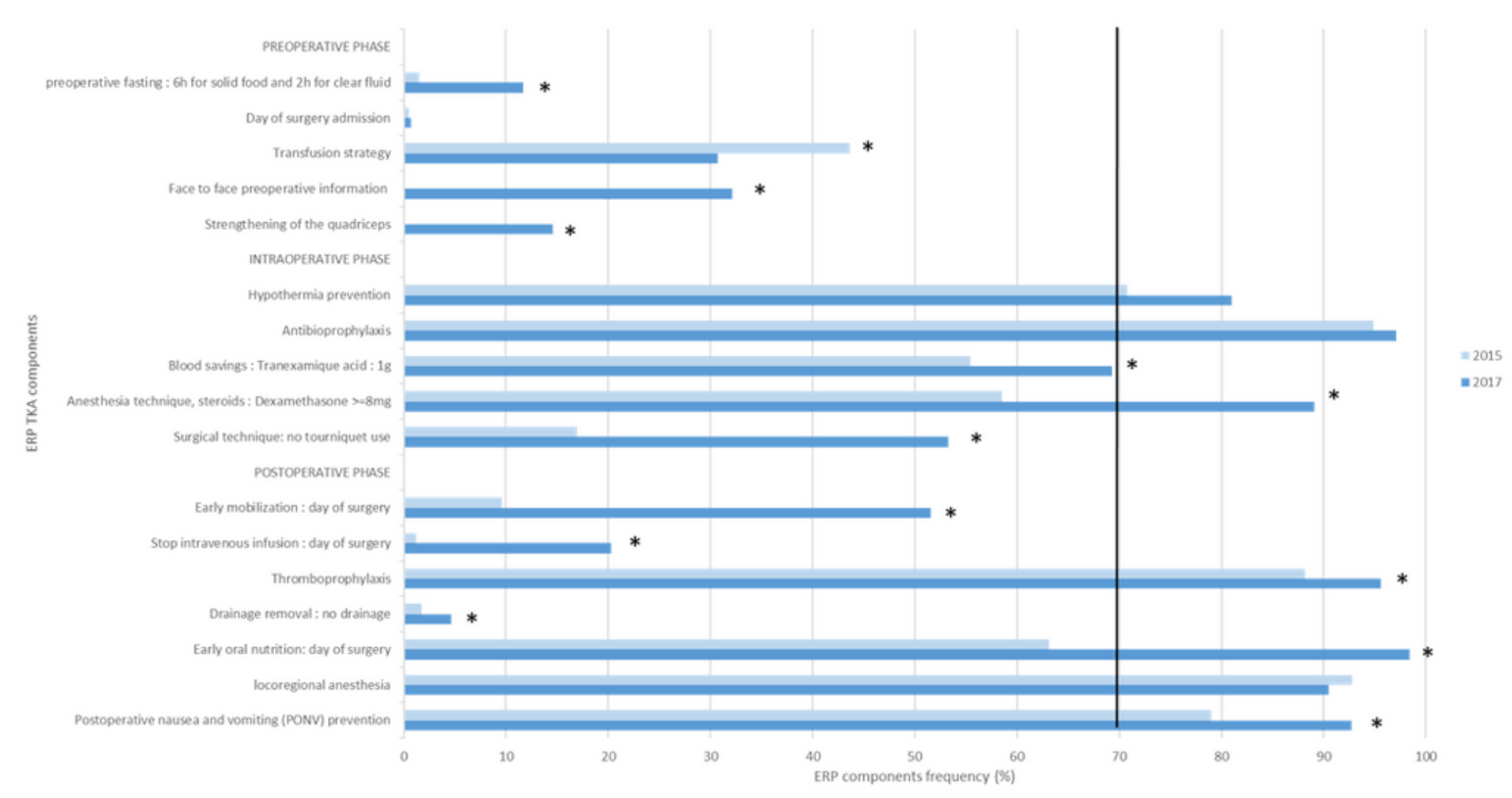

\section{Figure 1}


ERP components adherence in TKA patients in 2015 and 2017. Total knee arthroplasty (TKA) enhanced recovery program (ERP) components describing the patient management process pre, per and postoperatively were analyzed in frequency in data collected in 2015 and 2017 . The cut off value of $70 \%$ application was considered as reflecting sufficient appropriation. The frequency of ERP component application was compared with Chi2 test or Fischer test.

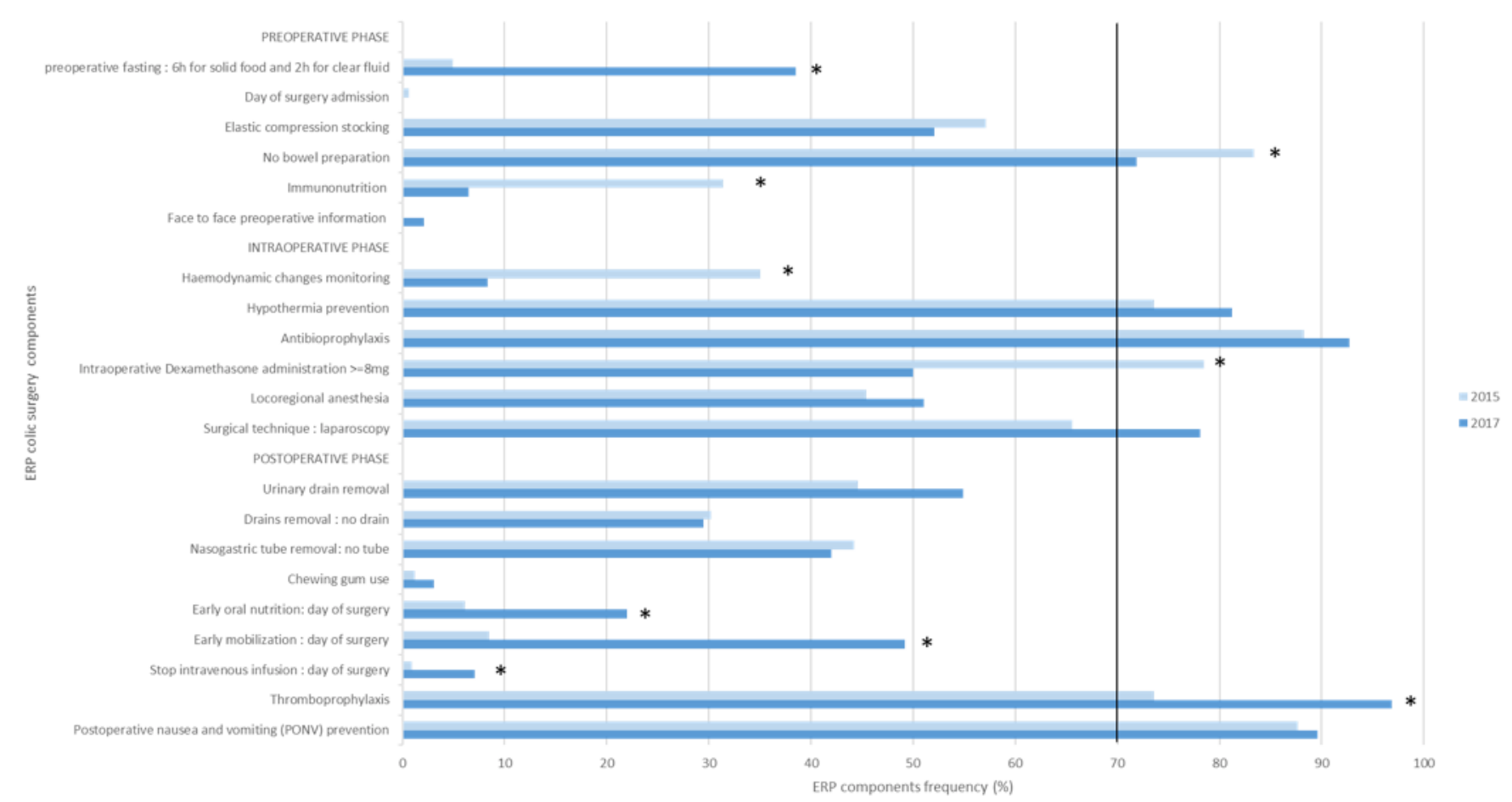

Figure 2

Comparison of frequency of ERP items application for colectomy in 2015 and 2017. Colectomy enhanced recovery program (ERP) components describing the patient management process pre, per and postoperatively was analyzed in frequency in data collected in 2015 and 2017 . The cut off value of $70 \%$ application was considered as reflecting sufficient appropriation. The frequency of ERP component application was compared with Chi2 test or Fischer test. 


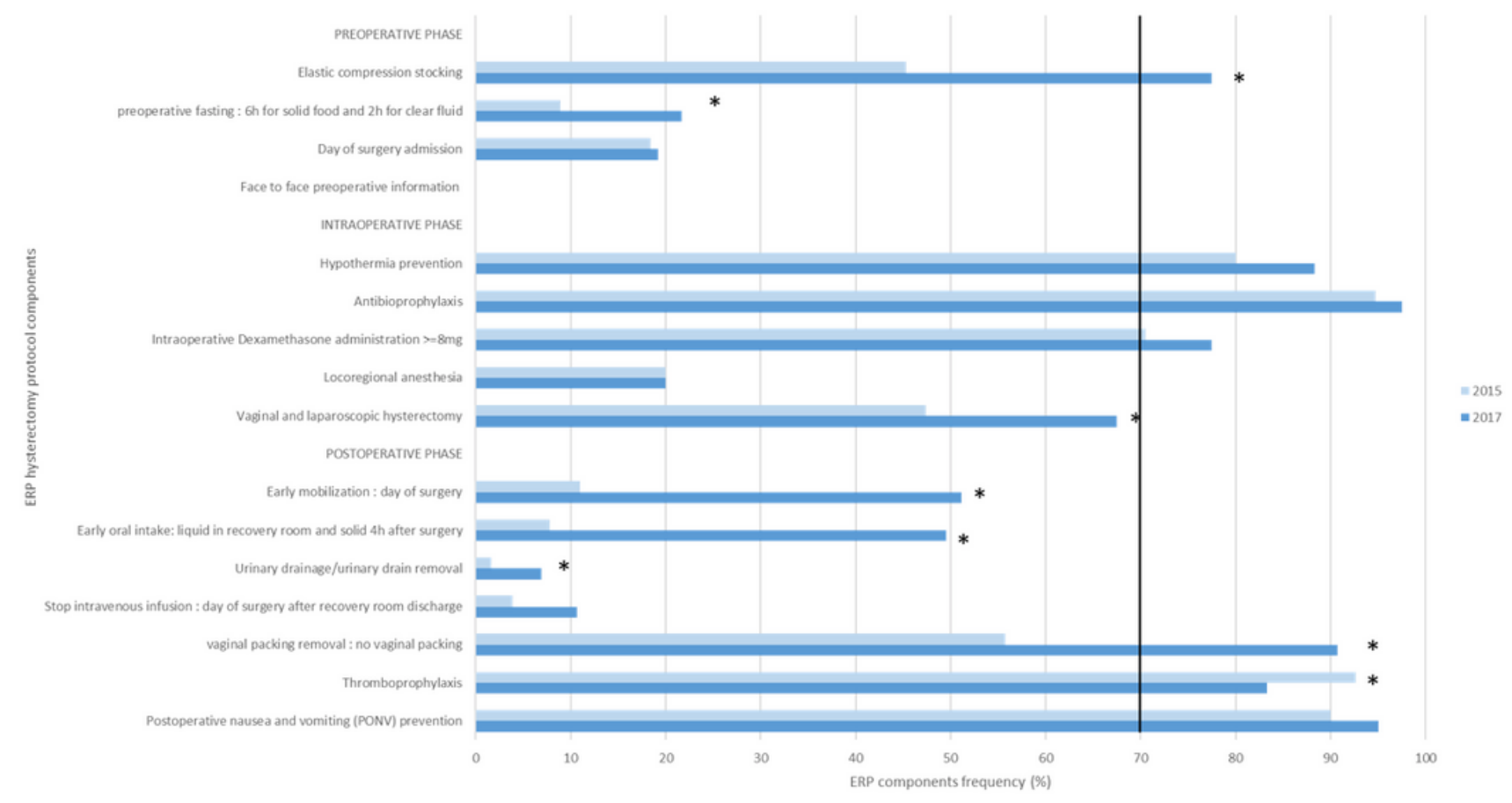

Figure 3

Comparison of frequency of ERP items application for hysterectomy in 2015 and 2017. Hysterectomy enhanced recovery program (ERP) components describing the patient management process pre, per and postoperatively was analyzed in frequency in data collected in 2015 and 2017 . The cut off value of $70 \%$ application was considered as reflecting sufficient appropriation. The frequency of ERP component application was compared with Chi2 test or Fischer test. 
A. Total knee arthroplasty

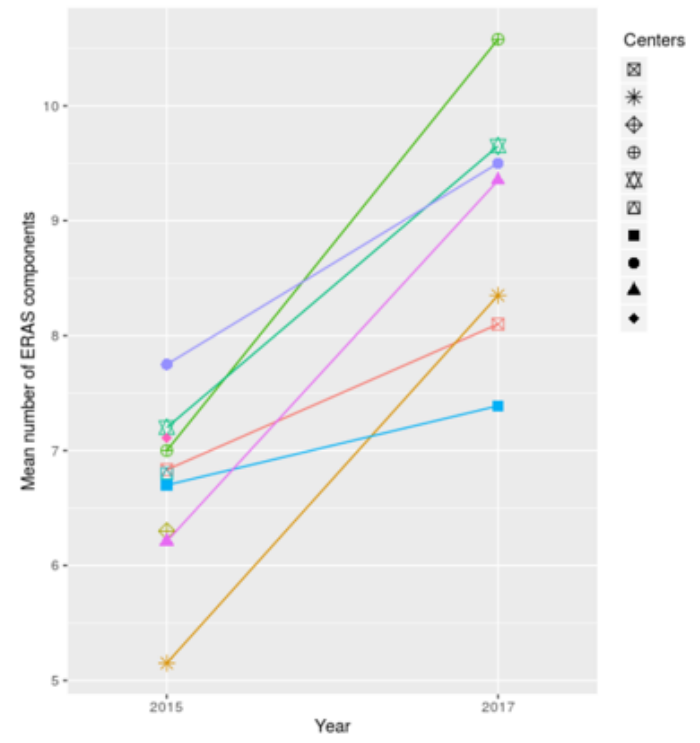

B. Colectomy

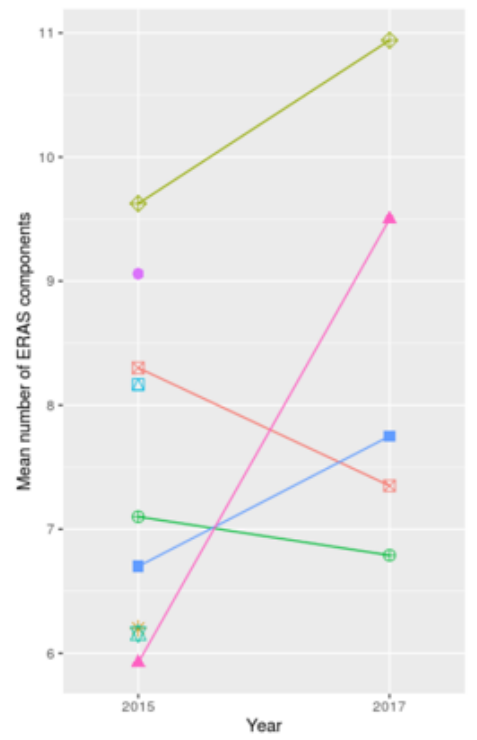

C. Hysterectomy

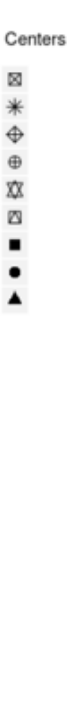

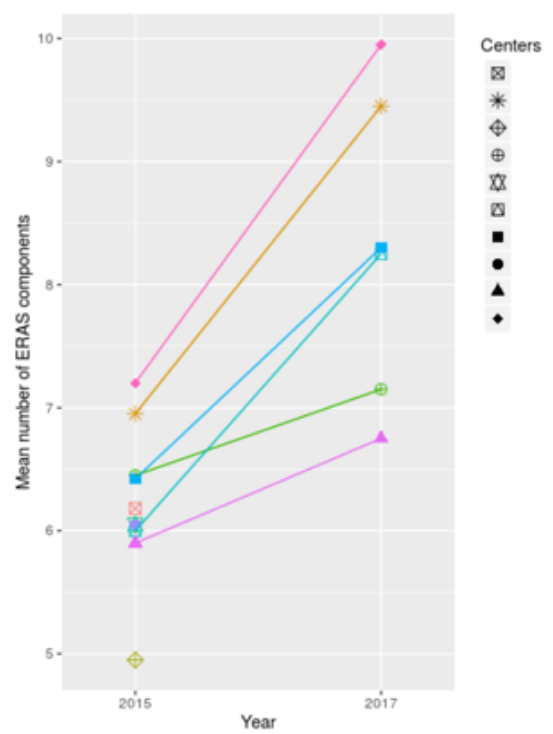

\section{Figure 4}

evolution of ERP items application in different centers. The frequency of ERP items application is described for all participating centers in 2015 and 2017 for the three surgical models total knee arthroplasty, colectomy and hysterectomy 


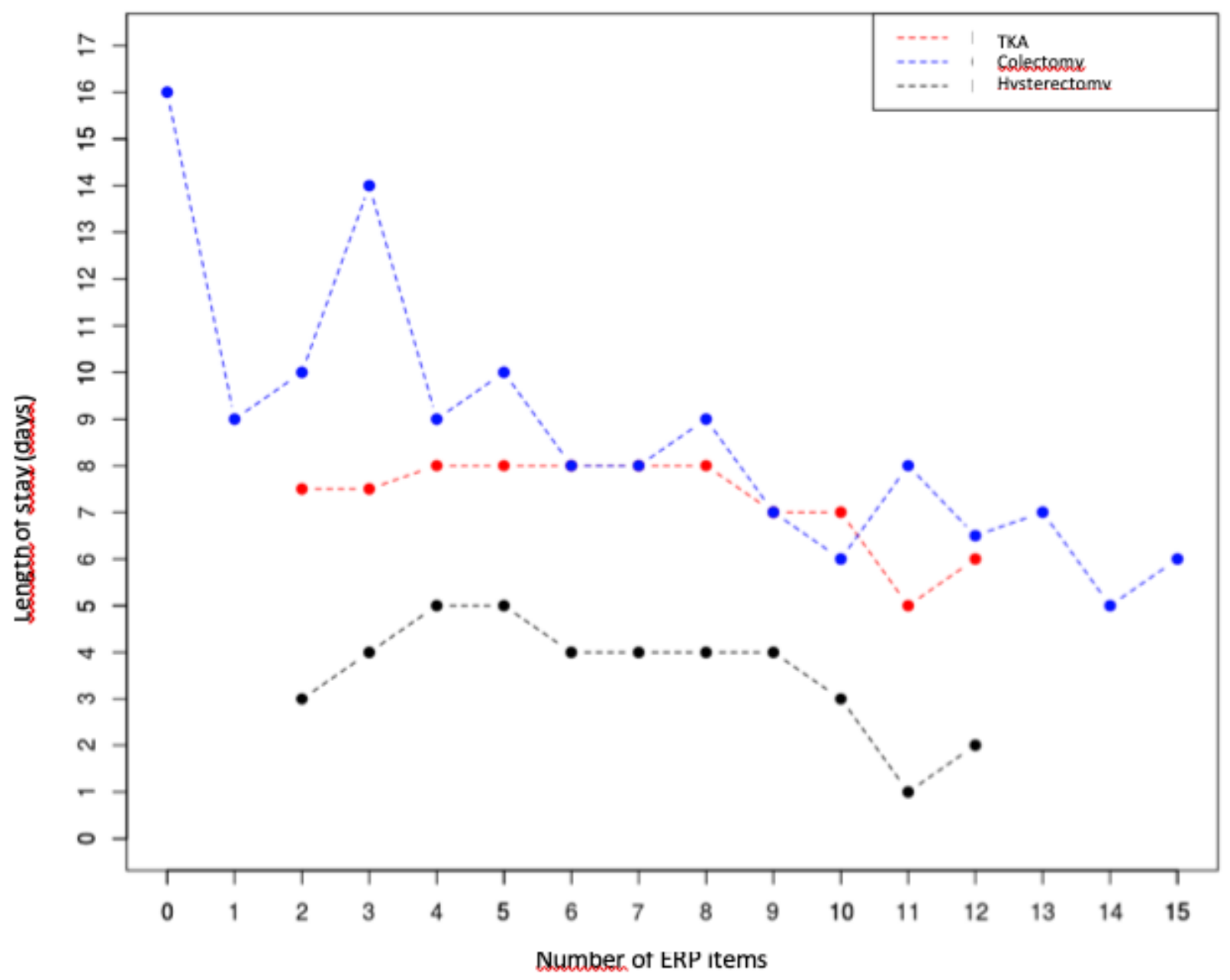

Figure 5

evolution of the median LOS depending of ERP items application after TKA, colectomy and hysterectomy. This figure describes the evolution of the median value of length of sty in the three surgical models total knee arthroplasty, colectomy and hysterectomy depending of ERP items application 
A. Total knee arthroplasty

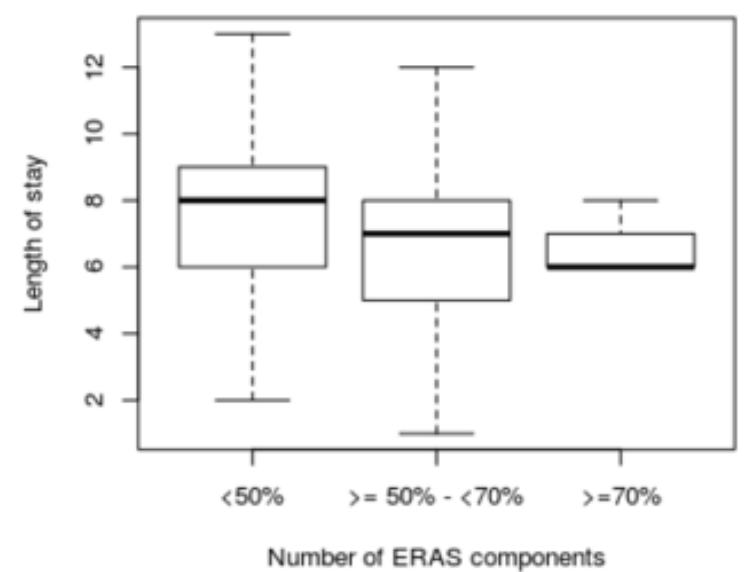

B. Colectomy

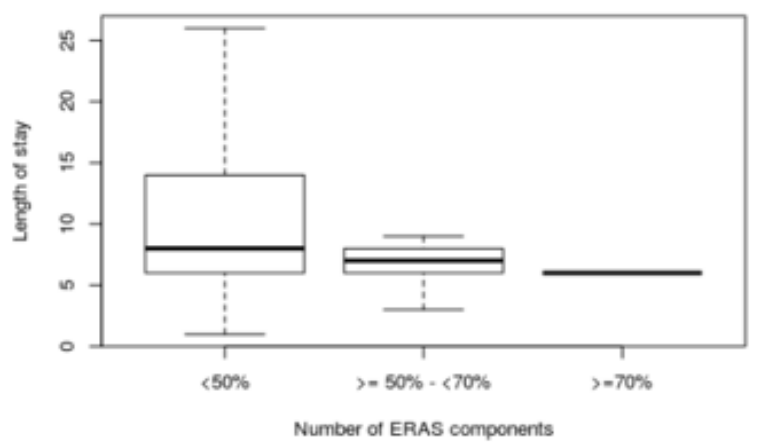

C. Hysterectomy

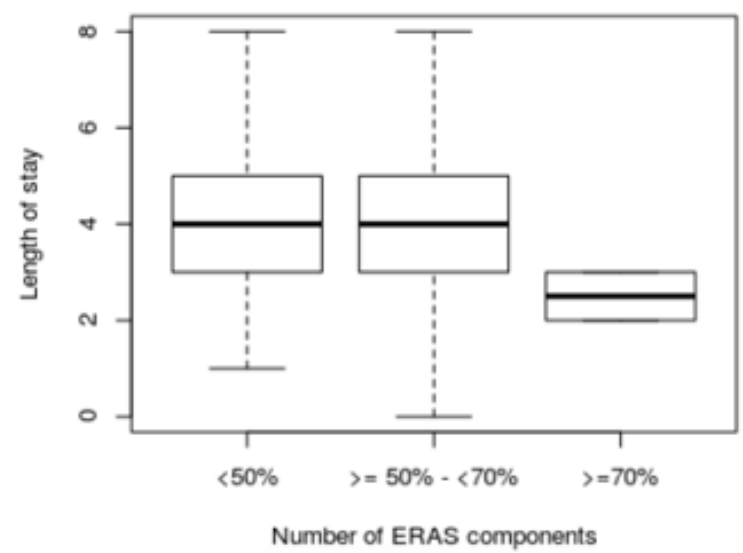

Figure 6

evolution of length of stay depending of the percentage of ERAS items application. This box plot figures describes all individual data without outliers collected in 2015 and 2017 for relation between individual LOS and number of ERP items applied grouped in three categories (i.e. ERP items application < 50\%; 50$70 \%,>70 \%$. There is a significant relation for TKA $(p=0.002)$ and hysterectomy $(p=0.002)$; Test de Kruskal Wallis). 